\title{
Renal Dysfunction Is Associated with Middle Cerebral Artery Pulsatility Index and Total Burden of Cerebral Small Vessel Disease
}

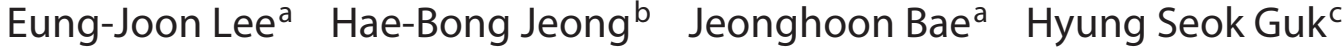 \\ Han-Yeong Jeong ${ }^{a}$ Eun Ji Lee ${ }^{d}$ Byung-Woo Yoon ${ }^{\text {a, e }}$
}

\begin{abstract}
${ }^{a}$ Department of Neurology, Seoul National University Hospital, Seoul, Republic of Korea; ${ }^{b}$ Department of Neurology, Chung-Ang University Hospital, Seoul, Republic of Korea; 'Department of Neurology, Gunsan Medical Center, Gunsan-si, Republic of Korea; 'Department of Radiology, Soonchunhyang University Seoul Hospital, Seoul, Republic of Korea; ' Department of Neurology, Uijeongbu Eulji Medical Center, Uijeongbu-si, Republic of Korea
\end{abstract}

\section{Keywords}

Stroke $\cdot$ Kidney disease $\cdot$ Vascular stiffness $\cdot$ Transcranial

Doppler ultrasonography · Cerebral small vessel disease

\begin{abstract}
Background and Purpose: Renal dysfunction is known to affect vasculature and lead to systemic arterial stiffness. It also independently increases the risk of cerebral small vessel disease (cSVD) and stroke. We aimed to examine the effect of renal dysfunction on cerebral hemodynamics and the burden of CSVD. Methods: Of the 412 patients admitted to Seoul National University Hospital, between May 2015 and 2019, with lacunar infarction and no major intracranial arterial stenosis observed on magnetic resonance angiography, we included 283 patients who had undergone a transcranial Doppler (TCD) ultrasound after $72 \mathrm{~h}$ of stroke onset. The patients were divided into renal dysfunction (estimated glomerular filtration rate $[e G F R]<60 \mathrm{~mL} / \mathrm{min} / 1.73 \mathrm{~m}^{2}$ at admission) and control (eGFR $\geq 60 \mathrm{~mL} / \mathrm{min} / 1.73 \mathrm{~m}^{2}$ ) groups. We investigated the correlations between renal function, the pulsatility index (PI), and the total MRI burden of cSVD. Furthermore, multivariate analysis was performed to assess the association between renal dysfunction and the PI of the middle cerebral artery (MCA) measured through TCD ultrasound. Results:
\end{abstract}

Among the total patients, 74 (26.1\%) had renal dysfunction (eGFR $<60 \mathrm{~mL} / \mathrm{min} / 1.73 \mathrm{~m}^{2}$ at admission). Patients with renal dysfunction were significantly older, showed higher pulse pressure, and had a higher prevalence of hypertension, diabetes mellitus, and coronary artery disease. Renal dysfunction was significantly associated with higher distal cerebrovascular flow resistance (median $\mathrm{PI} 1.12$, interquartile range [IQR]: 0.85-1.57 vs. controls 0.84, IQR: 0.54-1.22; $p<0.001$ ). Also, patients with renal dysfunction had a significantly higher total MRI burden of CSVD (median CSVD score 2, IQR: $1-3$ vs. controls median score 1, IQR: $0-2 ; p<0.001)$. There was an inverse proportional relationship between the PI and eGFR. Finally, multivariate analysis showed renal dysfunction (adjusted odds ratio: 4.516, 95\% confidence interval: 1.051-20.292) and older age (adjusted odds ratio: 1.076, 95\% confidence interval: 1.038-1.114) as independent predictors of a high PI. Conclusions: Renal dysfunction is independently associated with a high PI of MCA. Renal dysfunction leads to systemic arterial stiffness including stiffness in cerebral arteries, thus increasing the burden of cSVD. Therefore, noninvasive screening for high PI by TCD in kidney failure patients might be helpful.

(C) 2021 The Author(s)

Published by S. Karger AG, Basel

Eung-Joon Lee and Hae-Bong Jeong share first authorship. karger@karger.com www.karger.com/ced

Karger $\frac{1}{\%}$

BOPEN ACCESS
(C) 2021 The Author(s)

Published by S. Karger AG, Basel

This is an Open Access article licensed under the Creative Commons Attribution-NonCommercial-4.0 International License (CC BY-NC) (http://www.karger.com/Services/OpenAccessLicense), applicable to the online version of the article only. Usage and distribution for commercial purposes requires written permission.
Correspondence to:

Byung-Woo Yoon, bwyoon@snu.ac.kr 


\section{Introduction}

Poor renal function is known to affect the systemic vasculature and vascular remodeling, eventually leading to systemic arterial stiffness $[1,2]$. Arterial stiffening generally results from oxidative stress and chronic inflammation of the arterial wall, involving a variety of pathological factors of kidney disease, including uremic toxins [3]. Additionally, renal dysfunction increases the burden of cerebral small vessel disease (cSVD) and the risk of stroke $[4,5]$. The underlying causes of the progression of cSVD are poorly understood; however, the persistent pulsatile stress from systemic arterial stiffness has been suggested as a possible cause [6].

Transcranial Doppler (TCD) ultrasound is a noninvasive investigation used to obtain cerebral hemodynamic data, including Gosling's pulsatility index (PI). PI indicates the degree of downstream vascular resistance to flow. Aggravation of arterial stiffness decreases the damping of the vascular flow and increases PI of the cerebral blood flow [7]. Furthermore, it has been considered that increased PI of the middle cerebral artery (MCA) could be associated with cerebral arteriosclerosis, progression of white matter hyperintensities (WMHs), and cerebral atrophy $[8,9]$.

Therefore, we hypothesized that renal dysfunction affects systemic vasculature including cerebral arteries and is associated with high PI of MCA. To determine the effect of renal dysfunction on cerebral hemodynamics and burden of cSVD, this study aimed to analyze PI by kidney function. Moreover, we investigated the impacts of renal dysfunction on the total burden of cSVD.

\section{Materials and Methods}

\section{Participants}

In this retrospective study, we reviewed consecutive patients with acute ischemic stroke who had been admitted to Seoul National University Hospital between May 2015 and 2019. We identified 412 patients with lacunar infarcts without any major intracranial arterial stenosis or occlusion on magnetic resonance angiography. We defined lacunar infarction as a small infarct $(<20 \mathrm{~mm}$ on diffusionweighted image) in the territory of the perforating artery. Among these patients, 393 had undergone a TCD examination. Patients who had undergone TCD examinations $<72 \mathrm{~h}$ after ischemic stroke were excluded because of potential hemodynamic changes during the acute stage of ischemic stroke. We also excluded patients with atrial fibrillation or poor temporal insonation windows. Patients with internal carotid artery stenosis above $50 \%$ by The North American Symptomatic Carotid Endarterectomy Trial criteria were also excluded. In effect, the final study population was 283 . The study was approved by the Seoul National University Hospital Institutional Re- view Board. Informed consent was waived by the Institutional Review Board due to the retrospective nature of this study.

\section{Measurements}

We undertook a retrospective chart review to collect patient demographic and clinical data. TCD examinations were performed by experienced ultrasonographers at the Seoul National University Hospital. Both MCAs were examined at multiple depths using a transtemporal window. The PI was calculated using the following formula:

$\mathrm{PI}=($ peak systolic velocity - end diastolic velocity $) /$

mean flow velocity

We defined the PI of the bilateral MCAs as the mean of all measured PI values in both MCAs. Because the normal range of PI is 0.6-1.1, we defined high PI as above 1.1 [10].

Blood pressure and heart rate were measured immediately before the TCD examination using an automated blood pressure device. All evaluations were performed twice while the patient was sitting in a chair. Pulse pressure was calculated by subtracting the average diastolic blood pressure from the average systolic blood pressure. The heart rate of each patient was based on the average of the 2 measurements.

An experienced neurologist and a radiologist separately reviewed the results of brain MRI and were blinded to the clinical information and TCD findings. The definition of the standard criteria for markers for cSVD is based on international consensus [11]. We used the total cSVD score, composed of all 4 imaging markers of cSVD, to evaluate the total cSVD burden based on a recently developed scoring system [12-14]. One point was assigned for the presence of each marker: WMHs, with either irregular periventricular hyperintensities extending into the deep white matter (Fazekas score 3) and/or confluent deep WMHs (Fazekas score 2 or 3); a lacuna of $<20 \mathrm{~mm}$ in diameter, located in the internal or external capsule, basal ganglia, thalamus, or brain stem and not competed with the clinical symptoms; a deep cerebral microbleed located in the internal or external capsule, basal ganglia, or thalamus; and enlarged perivascular space with a scale of 2 or 3 in the basal ganglia, according to a 3-category ordinal scale $(0-10,10-25$, and $>25)$, in the hemisphere with the highest number of extensive perivascular spaces. Scores ranged from 0 to 4 and described the severity of the total cSVD burden.

\section{Statistical Analysis}

Means and medians were used as measurements of central tendencies as appropriate, with corresponding standard deviations and interquartile ranges, respectively. Comparisons between groups according to renal function were performed using Fisher's exact test for proportions. Continuous variables were analyzed using the Wilcoxon rank-sum test. The difference in total MRI burden of cSVD score and each feature of cSVD between renal dysfunction and control groups was analyzed. The correlation between the PI value and the total MRI burden of cSVD score was explored by linear regression analysis. For factors predicting the higher total MRI cSVD score, multiple linear regression was performed. We investigated the relationship between eGFR distribution and the PI to divide the eGFR into 5 categories. The eGFR levels were selected based on a chronic kidney disease classification in accordance with the National Kidney Foundation-Kidney Disease Outcomes Quality Initiative: eGFR $\geq 90$ $\mathrm{mL} / \mathrm{min}$ per $1.73 \mathrm{~m}^{2}$, eGFR $60-89 \mathrm{~mL} / \mathrm{min}$ per $1.73 \mathrm{~m}^{2}$, eGFR $30-59$ $\mathrm{mL} / \mathrm{min}$ per $1.73 \mathrm{~m}^{2}$, eGFR $15-29 \mathrm{~mL} / \mathrm{min}$ per $1.73 \mathrm{~m}^{2}$, and eGFR 
Table 1. Baseline characteristics of the patients according to kidney function

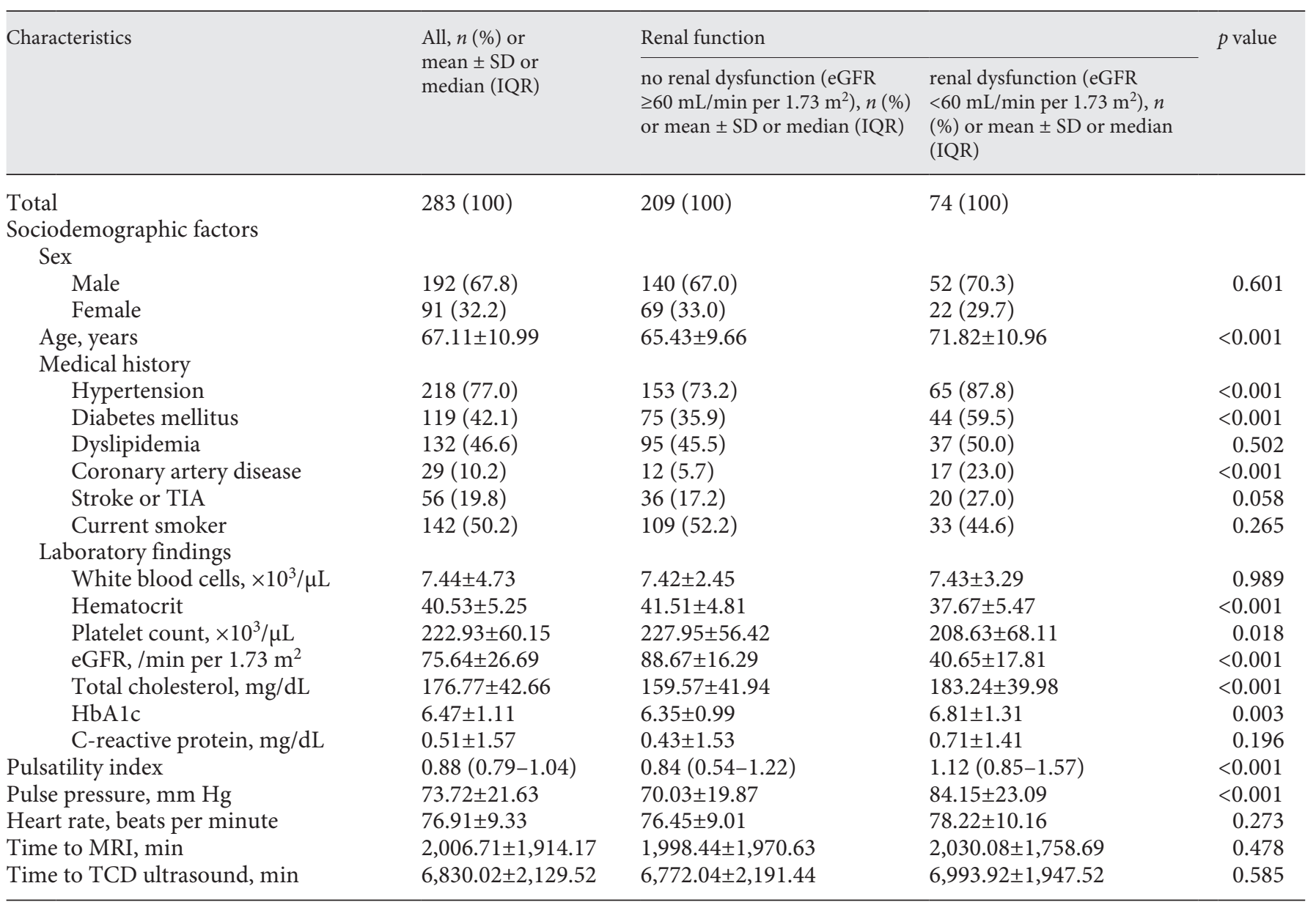

SD, standard deviation; IQR, interquartile range; TIA, transient ischemic attack; eGFR, estimated glomerular filtration rate; MDRD, Modification of Diet in Renal Disease; $\mathrm{mm} \mathrm{Hg}$, millimeter of mercury; MRI, magnetic resonance imaging; TCD, transcranial Doppler.

$<15 \mathrm{~mL} / \mathrm{min}$ per $1.73 \mathrm{~m}^{2}$. In this study, an eGFR $\geq 60 \mathrm{~mL} / \mathrm{min}$ per $1.73 \mathrm{~m}^{2}$ was classified as no renal dysfunction, and we selected patients with this eGFR as controls [15]. The diagnostic criteria of CKD require longitudinal eGFR data from at least 3 months, and because follow-up eGFRs were not available for all patients, we analyzed the data according to eGFR levels at admission rather than CKD stages. Finally, with the high PI (>1.1) as the dependent variable, multivariate models were constructed to examine whether renal dysfunction independently increased the PI. Statistical analysis was performed using IBM SPSS version 25 (IBM Corp., Armonk, NY, USA). A 2-sided $p$ value of $<0.05$ was considered statistically significant.

\section{Results}

\section{Baseline Characteristics of the Patients according to Kidney Function}

Of the 412 patients with acute lacunar infarction, 283 had undergone a TCD examination at least $72 \mathrm{~h}$ after stroke onset. The average time taken from the stroke onset to the TCD was $6,830.02 \pm 2,129.52 \mathrm{~min}$. Of these, 74 (26.1\%) had renal dysfunction (eGFR $<60 \mathrm{~mL} / \mathrm{min} / 1.73$ $\mathrm{m}^{2}$ at admission). Table 1 shows patient demographics, vascular comorbidities, and laboratory findings. Among the total patients, $67.8 \%$ were male. The mean age of total participants was $67.11 \pm 10.99$ years, whereas the mean ages of those without and those with renal dysfunction were $65.43 \pm 9.66$ and $71.82 \pm 10.96$ years, respectively. The patients with renal dysfunction were significantly older, showed higher level of pulse pressure (84.15 \pm $23.09 \mathrm{~mm} \mathrm{Hg}$ vs. control group $70.03 \pm 19.87 \mathrm{~mm} \mathrm{Hg}$, $p<0.001$ ), and had a higher prevalence of hypertension ( $87.8 \%$ vs. control group $73.2 \%, p<0.001$ ), diabetes mellitus (59.5\% vs. control group 35.9\%, $p<0.001$ ), and coronary artery disease $(23.0 \%$ vs. control group $5.7 \%, p<$ 


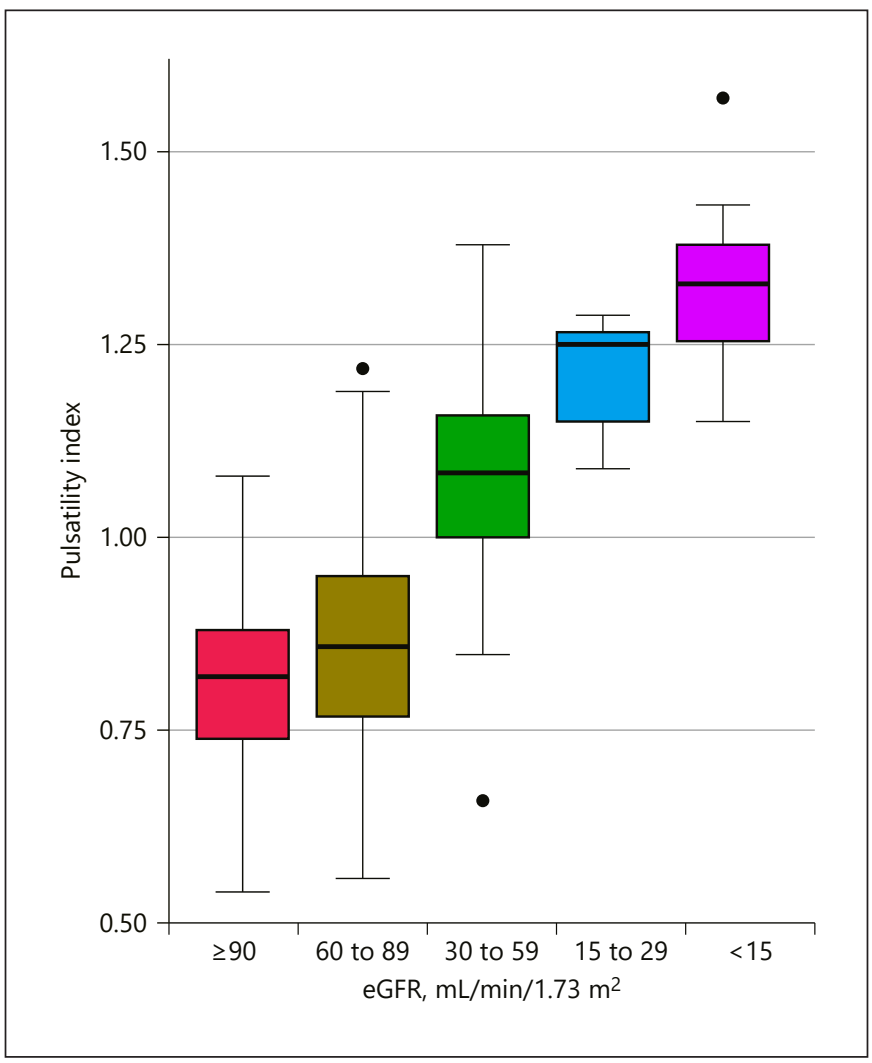

Fig. 1. Inverse proportional correlation between eGFR and pulsatility index. eGFR, estimated glomerular filtration rate.

0.001) than the control group. However, hematocrit $(37.67 \pm 5.47 \%$ vs. control $41.51 \pm 4.81 \%, p<0.001)$ and platelet count $\left(208.63 \pm 68.11 \times 10^{3} / \mu \mathrm{L}\right.$ vs. control 227.95 $\pm 56.42 \times 10^{3} / \mu \mathrm{L}, p=0.018$ ) were significantly lower in the renal dysfunction group. Renal dysfunction was significantly associated with higher arterial stiffness (median PI 1.12, interquartile range [IQR]: $0.85-1.57$ vs. controls median PI 0.84, IQR: $0.54-1.22 ; p<0.001$ ) of MCA. Furthermore, a proportional relationship was found between the degree of renal dysfunction and PI (shown in Fig. 1).

\section{The Total Burden of cSVD according to Renal Function}

The total MRI cSVD score was calculated using a previously explained 4 -point scale. Each 1 point was assigned for the presence of each of the following: the presence of 1 or more lacunes, presence of cerebral microbleeds, moderate to severe basal ganglia PVSs, and WMHs. Patients with renal dysfunction (eGFR $<60 \mathrm{~mL} / \mathrm{min}$ per 1.73 $\mathrm{m}^{2}$ ) had a significantly higher cSVD burden than the con- trol group (renal dysfunction group median: 2, IQR: 1-3; control group median: 1 , IQR: $0-2, p<0.001)$. All kinds of cSVDs, lacunae, cerebral microbleeds, extensive perivascular spaces, and WMHs, were observed significantly more frequently in patients with renal dysfunction. In the linear regression analysis, the total MRI burden of cSVD was significantly associated with MCA PI $(p<0.001$, see Table 2). Besides, in the multiple linear regression analysis, old age $(\beta 0.601$, standard error $[\mathrm{SE}] 0.005, p<0.001)$ and renal dysfunction $(\beta 0.226$, SE $0.114, p<0.001)$ were significantly correlated with total MRI cSVD score (Table 3).

\section{Factors Associated with High Intracranial Vascular}

\section{Resistance}

A stepwise multivariate linear regression analysis was performed to identify independent predictors of the high PI (>1.1). Risk factors known to affect arterial stiffness, such as older age, hypertension, diabetes mellitus, dyslipidemia, coronary artery disease, previous history of stroke or transient ischemic attack, smoking, and pulse pressure, were included. We also included hematocrit as a variable considering that blood viscosity may affect the result of TCD. Renal dysfunction (adjusted odds ratio [aOR]: 4.516, 95\% confidence interval [CI]: 1.051-20.292) and older age (aOR: 1.076, 95\% CI: 1.038-1.114) were independent predictors of a high PI in the multivariate model (Table 4).

\section{Discussion}

Renal dysfunction is known to affect systemic vasculature and lead to arterial stiffness caused by various uremic toxins. Earlier studies showed a gradual decline in renal excretion causing an increase in serum phosphates. Increased phosphate levels may induce vascular calcification directly through activation of proinflammatory molecules that are signaled in vascular smooth muscle cells [16]. Uric acid, another vascular toxin, is increased in patients with renal dysfunction. Uric acid decreases nitric oxide production by impeding the activity of endothelial nitric oxide synthase, which facilitates the proliferation of vascular smooth muscle cells [17]. Last, hypertriglyceridemia is frequently found in patients with renal dysfunction [18]. This condition can trigger oxidative stress, vascular inflammation, foam cell formation, and endothelial dysfunction [19].

Previous studies have suggested that renal dysfunction independently predicts the presence of cSVD [5]. 
Table 2. MRI feature and total burden of $\mathrm{CSVD}^{+}$according to renal function

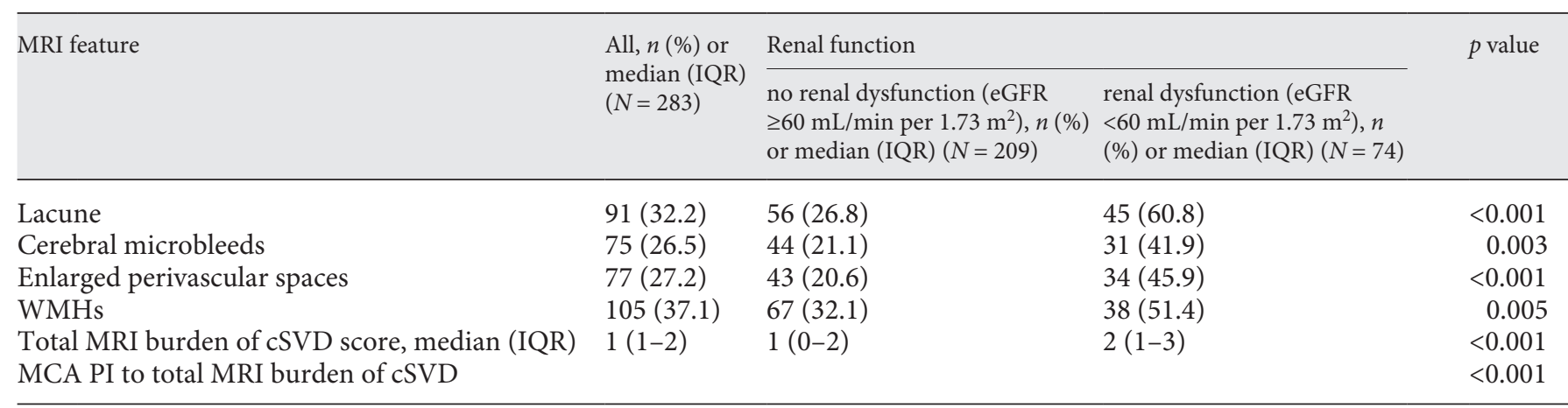

cSVD, cerebral small vessel disease; MRI, magnetic resonance imaging; IQR, interquartile range; eGFR, estimated glomerular filtration rate; cSVD, cerebral smallvessel disease; MCA, middle cerebral artery; PI, pulsatility index; WMHs, white matter hyperintensities. tWMHs defined as either irregular periventricular hyperintensities extending into the deep white matter (Fazekas score 3) and/or confluent deep WMHs (Fazekas score 2 or 3); a lacune, located in the internal or external capsule, basal ganglia, thalamus, or brain stem with a diameter $<20 \mathrm{~mm}$ and not competed with the clinical symptoms; cerebral microbleeds located in the internal or external capsule, basal ganglia, or thalamus; and enlarged perivascular space in the basal ganglia with a scale of 2 or 3, pursuant to a 3-category ordinal scale $(0-10,10-25$, and $>25)$, in the hemisphere with the highest number of extensive perivascular spaces.

Table 3. Factors associated with total MRI burden of cSVD

\begin{tabular}{|c|c|c|c|c|c|c|c|c|}
\hline \multirow[t]{2}{*}{ Factors } & \multicolumn{2}{|c|}{$\begin{array}{l}\text { Unstandardized } \\
\text { coefficients }\end{array}$} & \multirow{2}{*}{$\begin{array}{l}\text { Standardized } \\
\text { coefficients } \\
\beta\end{array}$} & \multirow[t]{2}{*}{$t$} & \multirow[t]{2}{*}{$p$ value } & \multicolumn{2}{|c|}{$95 \%$ CI for $B$} & \multirow[t]{2}{*}{ VIF } \\
\hline & $B$ & SE & & & & $\begin{array}{l}\text { lower } \\
\text { bound }\end{array}$ & $\begin{array}{l}\text { upper } \\
\text { bound }\end{array}$ & \\
\hline Age & 0.061 & 0.005 & 0.601 & 13.308 & $<0.001$ & 0.052 & 0.070 & 1.165 \\
\hline Renal dysfunction $\left(\mathrm{eGFR}<60 \mathrm{~mL} / \mathrm{min}\right.$ per $\left.1.73 \mathrm{~m}^{2}\right)$ & 0.568 & 0.114 & 0.226 & 4.973 & $<0.001$ & 0.343 & 0.793 & 1.181 \\
\hline Hypertension & 0.088 & 0.112 & 0.033 & 0.033 & 0.440 & -0.136 & 0.311 & 1.069 \\
\hline Diabetes mellitus & 0.059 & 0.097 & 0.027 & 0.611 & 0.541 & -0.132 & 0.251 & 1.078 \\
\hline Dyslipidemia & 0.013 & 0.096 & 0.006 & 0.134 & 0.893 & -0.176 & 0.202 & 1.076 \\
\hline Coronary artery disease & 0.043 & 0.162 & 0.012 & 0.265 & 0.791 & -0.275 & 0.361 & 1.127 \\
\hline Previous stroke or TIA & 0.236 & 0.118 & 0.086 & 2.003 & 0.062 & -0.011 & 0.458 & 1.060 \\
\hline Current smoker & 0.017 & 0.095 & 0.007 & 0.173 & 0.863 & -0.171 & 0.204 & 1.062 \\
\hline
\end{tabular}

cSVD, cerebral small vessel disease; MRI, magnetic resonance imaging; eGFR, estimated glomerular filtration rate; TIA, transient ischemia attack; CI, confidence interval; VIF, variance inflation factor; D-W, Durbin-Watson statistic. $F=39.415(p<0.001), R^{2}=0.536$, adjusted $R^{2}=0.522, \mathrm{D}$-W 1.565 .

Central arterial stiffness, such as aortic stiffening, transmits potentially harmful pulsatile energy to distal vessels [20]. Increased transmission of this energy into peripheral arteries causes reactive changes in distal vascular resistance to flow as well. If this is maintained over a long duration, it will lead to remodeling of the vessel wall composition and permanent changes in the distal vasculature [21]. Therefore, this mechanism is considered to have the pathophysiological potential to progression of cSVD.
The results of our study support those from previous studies that renal dysfunction causes stiffness of systemic vasculature and leads to the progression of cSVD. Furthermore, we demonstrated an inverse proportional relationship between the aggravation of renal dysfunction and the increase of PI. Thus, our results show that the progression of cSVD might be due to aggravation of systemic and cerebral arterial stiffness caused by renal dysfunction. Because the presence of cSVD is known to be associated with a higher risk of stroke and its unfavorable outcomes, these data provide evidence for the need to as- 
Table 4. Factors associated with high intracranial vascular resistance $(\mathrm{PI}>1.1)$

\begin{tabular}{|c|c|c|}
\hline \multirow[t]{2}{*}{ Factors } & \multicolumn{2}{|c|}{$\begin{array}{l}\text { Multivariate logistic regression } \\
\text { analysis }(n=283)\end{array}$} \\
\hline & adjusted OR* (95\% CI) & $p$ value \\
\hline Renal dysfunction $\left(e G F R<60 \mathrm{~mL} / \mathrm{min}\right.$ per $1.73 \mathrm{~m}^{2}$ ) & $4.116(1.013-19.292)$ & $<0.001$ \\
\hline Age & $1.025(0.980-1.071)$ & 0.017 \\
\hline Female gender & $0.680(0.233-1.980)$ & 0.479 \\
\hline Hypertension & $0.858(0.252-2.919)$ & 0.806 \\
\hline Diabetes mellitus & $1.196(0.485-2.950)$ & 0.698 \\
\hline Dyslipidemia & $1.004(0.405-2.485)$ & 0.994 \\
\hline Coronary artery disease & $1.028(0.339-3.114)$ & 0.961 \\
\hline Previous stroke or TIA & $1.541(0.575-4.129)$ & 0.389 \\
\hline Current smoker & $0.595(0.221-1.602)$ & 0.304 \\
\hline Pulse pressure & $1.010(0.990-1.031)$ & 0.321 \\
\hline Heart rate & $1.025(0.980-1.071)$ & 0.287 \\
\hline Hematocrit & $0.827(0.671-1.019)$ & 0.075 \\
\hline
\end{tabular}

eGFR, estimated glomerular filtration rate; OR, odds ratio; TIA, transient ischemia attack; CI, confidence interval. * Adjusted for sex, age, renal dysfunction, history of hypertension, diabetes mellitus, dyslipidemia, coronary artery disease, smoking status, previous ischemic stroke or TIA, pulse pressure, and hematocrit. sess the risk of ischemic stroke using TCD in patients with kidney failure and for early intervention addressing risk factors for ischemic stroke.

A strength of this study is that the PI was accurately evaluated by excluding any stenosis or occlusion in the cerebral arteries. Considering hemodynamic changes that occur in the acute stroke phase, we did not include TCD data that had been obtained $<72 \mathrm{~h}$ from symptom onset time. In addition to those risk factors related to arterial stiffness, we adjusted for hematocrit, which may affect the results of TCD. Furthermore, we showed a consistent trend that the PI of MCA is inversely proportional to the eGFR measured at admission. This has not been reported in any previous study.

This study had some limitations. First, it was a singlecenter, retrospectively designed study, and the sample size was relatively small. Second, we did not assess all medical conditions such as cerebral edema which may influence the PI. However, we only included lacunar stroke patients, and no patient had a craniectomy. Therefore, it is assumed that the effect of cerebral edema was insignificant in this study. Last, we defined the renal dysfunction from a single examination performed at admission. A follow-up evaluation of renal function was not performed in all patients. Renal dysfunction might be overdiagnosed due to concomitant medical conditions such as dehydration related to acute stroke. Also, data were cross-sectional, and we were not able to address longitudinal relationships be- tween the aggravation of renal dysfunction and changes in cerebral hemodynamics. Further, large-scale studies considering these factors will be needed to validate our results.

\section{Conclusion}

Renal dysfunction is known to affect the vasculature and eventually leads to systemic arterial stiffness. Pulsatile stress from arterial stiffness is known to be associated with the progression of cSVD. We demonstrated that renal dysfunction is independently associated with a high PI and correlates with the total burden of cSVD. Noninvasive screening for CSVD by TCD in kidney failure patients might be helpful.

\section{Statement of Ethics}

The study was approved by the Seoul National University Hospital Institutional Review Board (IRB No. 1009-062-332) and was conducted in accordance with ethical standards stated in the Declaration of Helsinki, 1983. Informed consent was waived by the Institutional Review Board due to the retrospective nature of this study.

\section{Conflict of Interest Statement}

The authors have no conflicts of interest to declare. 


\section{Funding Sources}

This research was supported by a fund (\#2020ER620200) by the Korea Centers for Disease Control \& Prevention, Republic of Korea.

\section{References}

1 Briet M, Bozec E, Laurent S, Fassot C, London GM, Jacquot C, et al. Arterial stiffness and enlargement in mild-to-moderate chronic kidney disease. Kidney Int. 2006;69(2):350-7.

2 Briet M, Boutouyrie P, Laurent S, London GM. Arterial stiffness and pulse pressure in CKD and ESRD. Kidney Int. 2012;82(4):388400.

3 Garnier A-S, Briet M. Arterial stiffness and chronic kidney disease. Pulse. 2015;3(3-4): 229-41.

4 Dad T, Weiner DE. Stroke and chronic kidney disease: epidemiology, pathogenesis, and management across kidney disease stages. Semin Nephrol. 2015;35:311-22.

5 Toyoda K. Cerebral small vessel disease and chronic kidney disease. J Stroke. 2015;17(1): 31.

6 Moore SE, Stabeno PJ, Van Pelt TI. The synthesis of arctic research (SOAR) project. Deep Sea Res. 2018;152:1-7.

7 Robba C, Goffi A, Geeraerts T, Cardim D, Via G, Czosnyka M, et al. Brain ultrasonography: methodology, basic and advanced principles and clinical applications. A narrative review. Intensive Care Med. 2019;45:913-27.

8 Aribisala BS, Morris Z, Eadie E, Thomas A, Gow A, Valdés Hernández MC, et al. Blood pressure, internal carotid artery flow parameters, and age-related white matter hyperintensities. Hypertension. 2014;63(5):1011-8.

\section{Author Contributions}

E. Lee and H.B. Jeong conceived and designed the study, acquired and analyzed the data, interpreted the study findings, and drafted the manuscript. E.J. Lee analyzed data. H. Jeong and J. Bae supervised and directed the conduct of the study, interpreted the study findings, and critically revised the manuscript. B. Yoon critically reviewed the manuscript. All authors had full access to all of the data and the accuracy of the data analysis.
9 Purkayastha S, Fadar O, Mehregan A, Salat DH, Moscufo N, Meier DS, et al. Impaired cerebrovascular hemodynamics are associated with cerebral white matter damage. J Cereb Blood Flow Metab. 2014;34(2):228-34.

10 Aaslid R, Lindegaard K-F. Cerebral hemodynamics. Transcranial Doppler sonography: Springer; 1986. p. 60-85

11 Wardlaw JM, Smith EE, Biessels GJ, Cordonnier C, Fazekas F, Frayne R, et al. Neuroimaging standards for research into small vessel disease and its contribution to ageing and neurodegeneration. Lancet Neurol. 2013; 12(8):822-38

12 Huijts M, Duits A, Van Oostenbrugge RJ, Kroon AA, De Leeuw PW, Staals J. Accumulation of MRI markers of cerebral small vessel disease is associated with decreased cognitive function. A study in first-ever lacunar stroke and hypertensive patients. Front Aging Neurosci. 2013;5:72.

13 Klarenbeek P, van Oostenbrugge RJ, Rouhl RP, Knottnerus IL, Staals J. Ambulatory blood pressure in patients with lacunar stroke: association with total MRI burden of cerebral small vessel disease. Stroke. 2013;44(11): 2995-9.

14 Staals J, Booth T, Morris Z, Bastin ME, Gow AJ, Corley J, et al. Total MRI load of cerebral small vessel disease and cognitive ability in older people. Neurobiol Aging. 2015;36(10): 2806-11.
15 Levey AS, Coresh J, Balk E, Kausz AT, Levin A, Steffes MW, et al. National kidney foundation practice guidelines for chronic kidney disease: evaluation, classification, and stratification. Ann Intern Med. 2003;139(2):137-47.

16 Shanahan CM, Crouthamel MH, Kapustin A, Giachelli CM. Arterial calcification in chronic kidney disease: key roles for calcium and phosphate. Circ Res. 2011;109(6):697-711.

17 Six I, Flissi N, Lenglet G, Louvet L, Kamel S, Gallet $\mathrm{M}$, et al. Uremic toxins and vascular dysfunction. Toxins. 2020;12(6):404.

18 Tsimihodimos V, Mitrogianni Z, Elisaf M. Dyslipidemia associated with chronic kidney disease. Open Cardiovasc Med J. 2011;5:41.

19 Mikolasevic I, Žutelija M, Mavrinac V, Orlic L. Dyslipidemia in patients with chronic kidney disease: etiology and management. Int J Nephrol Renovasc Dis. 2017;10:35.

20 de Roos A, van der Grond J, Mitchell G, Westenberg J. Magnetic resonance imaging of cardiovascular function and the brain: is dementia a cardiovascular-driven disease? Circulation. 2017;135(22):2178-95.

21 Kim MO, Li Y, Wei F, Wang J, O'Rourke MF, Adji A, et al. Normal cerebral vascular pulsations in humans: changes with age and implications for microvascular disease. J Hypertens. 2017;35(11):2245-56. 